\title{
Spontaneous Retropharyngeal Haematoma in a One-Year-Old Child: A Case Report
}

\author{
R. K. Mundra*, Richi Sinha, Sunil Bajoliya \\ Department of Otorhinolaryngology Head and Neck Surgery, MGM Medical College \& MY Hospitals, \\ Indore, India \\ Email: *mundra.rk@gmail.com
}

Received 15 March 2014; revised 14 April 2014; accepted 11 May 2014

Copyright (C) 2014 by authors and Scientific Research Publishing Inc.

This work is licensed under the Creative Commons Attribution International License (CC BY). http://creativecommons.org/licenses/by/4.0/

c) (i) Open Access

\begin{abstract}
Spontaneous retropharyngeal haematoma is an unusual condition and difficult to diagnosis early especially in infants. It has multiple aetiological factors and can present to a number of specialists including the otolaryngologist. We describe an unusual case of a spontaneous huge retropharyngeal haematoma in 1-year-old male child which demonstrates the dramatic presentation and emphasizes the need for immediate surgical approach.
\end{abstract}

\section{Keywords}

\section{Cervical, Haematoma, Prevertebral, Retropharyngeal}

\section{Introduction}

Retropharyngeal haematoma is a life-threatening emergency with multiple aetiological factors [1]. Although mostly seen in adults, spontaneous retropharyngeal haematoma is an unusual condition and difficult to diagnosis early especially in infants. It can present to a number of specialists including the otolaryngologist and should be anticipated in all cervical spine injured patients regardless of the severity of trauma, contact sports injuries, blow to the head from falling object or a punch and shaken baby syndrome. Most retropharyngeal haematomas described in the literature have involved complicating factors such as anticoagulant therapy, tumour, aneurysm, infection [1] [2]. We describe an unusual case of spontaneous huge retropharyngeal haematoma in 1-year-old male child which demonstrates the dramatic presentation and emphasises the need for immediate surgical approach.

"Corresponding author. 


\section{Case Report}

\subsection{Patient History}

A 1-year-old male child was admitted to the Emergency Unit of the hospital; the mother informed that the child had been feverish for the past 10 days, and that he was suffering from cough and cold. There was no specific past medical history such as trauma, administration of anticoagulants or any other medical condition. She had sought a clinician locally, and the child had been prescribed amoxicillin which was taken for 2 days. After four days, the child developed difficulty in breathing and swallowing; with the appearance of right side neck swelling. He was drowsy and refused feeds.

\subsection{Physical Examination}

The child was fatigued, febrile, tachycardic and tachypneic with inspiratory stridor. There was a diffuse tender swelling of about $3 \times 2.5 \mathrm{~cm}$ over right side of submandibular region, the right contour of the neck and the posterior oropharyngeal wall were altered. Oral intubation was done promptly, blood samples were taken for a complete blood count and culture showing markedly raised total leucocyte count, and intravenous antibiotics (amoxicillin + clavulanate and metronidazole) were started.

\subsection{Radiography}

Computed tomography of the neck revealed mixed attenuating solid to cystic collection in retropharyngeal and right parapharyngeal space extending from nasopharynx to mid neck of size $3.6 \times 4.5 \times 5.1 \mathrm{~cm}$ likely a retropharyngeal abscess or haemorrhagic (Figure 1 and Figure 2). There was also lateral displacement of right common carotid and internal jugular vein, with obscuring of part of carotid vessel by the collection which may be suggestive of some possible injury to the vessel.

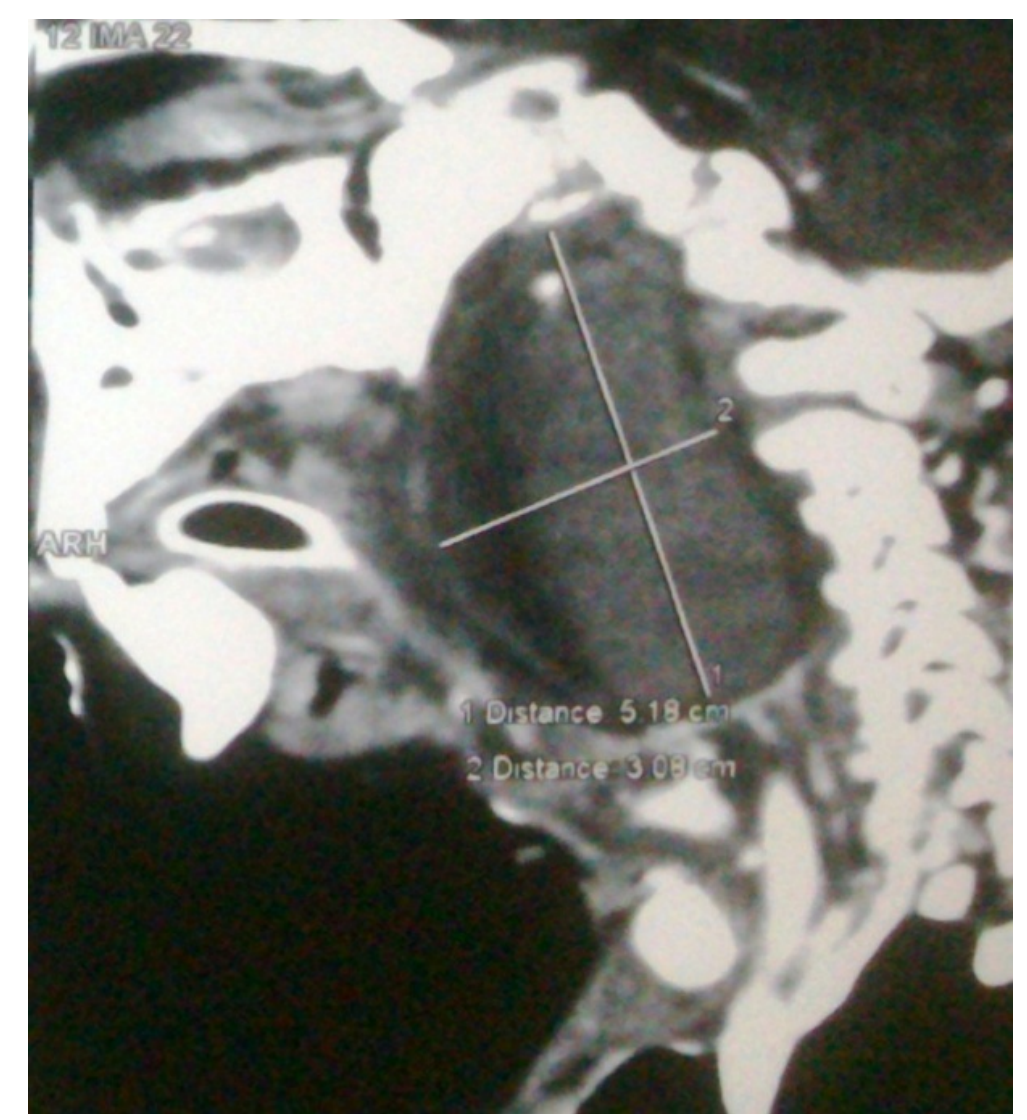

Figure 1. CT scan lateral view showing solid to cystic collection in retropharyngeal and right parapharyngeal space. 


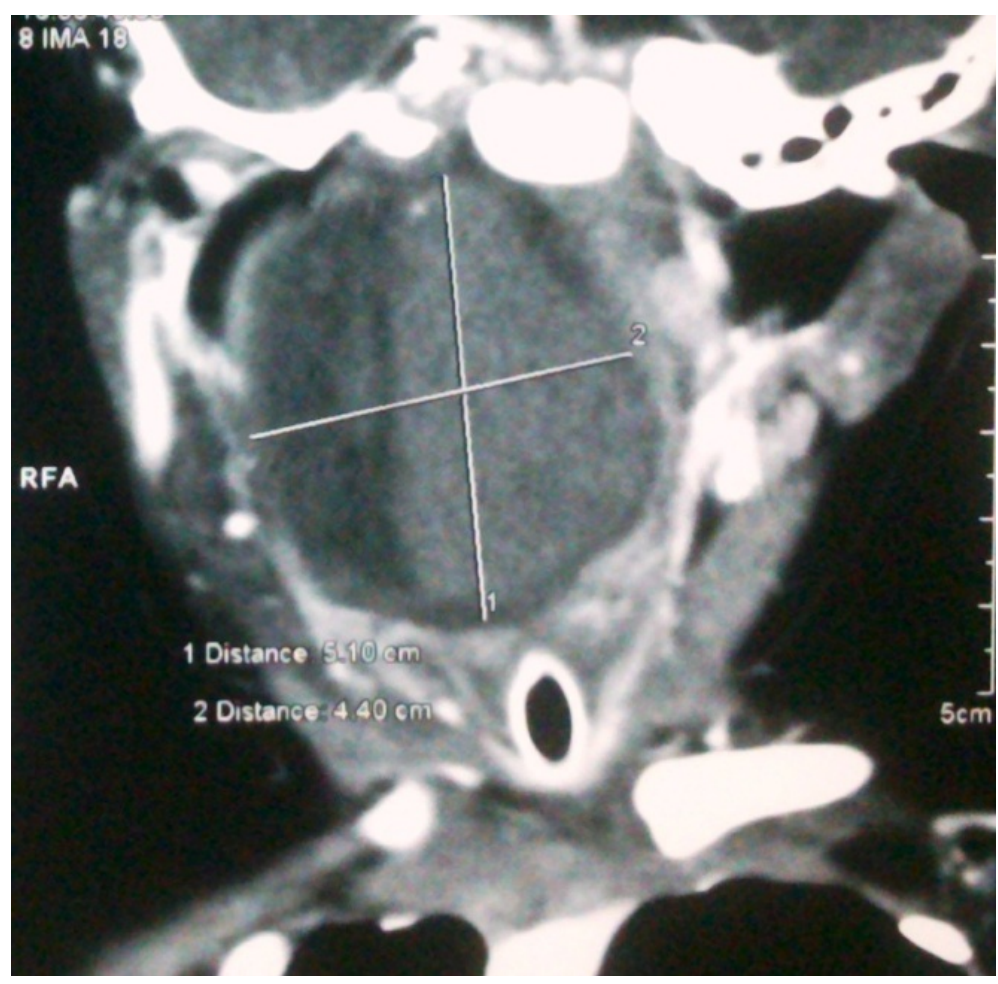

Figure 2. CT scan of same patient in coranal view.

\subsection{Management}

There was spontaneous rupture of the swelling with throat bleed. Immediate surgical drainage was achieved by a transoral approach with evacuation of all the blood clots. There was no pus seen intraoperatively indicating a retropharyngeal hematoma. In spite of adequate drainage, the child had already suffered from hypoxic injuries and had grave prognosis.

\section{Discussion}

Retropharyngeal hematoma is rare, difficult to diagnose early, and may progress to airway obstruction.

\subsection{Etiology}

Etiologies of retropharyngeal hematoma include infection, cervical spine trauma, great vessel trauma, violent head movements, iatrogenic injury associated with cardiac catheterization, cerebral angiography, parathyroid adenoma hemorrhage, and foreign body ingestion. Anticoagulation or hemorrhagic diathesis predisposes an individual to develop retropharyngeal hematoma. Spontaneous retropharyngeal hematoma is defined by the absence of any clear etiology, and there are only a few case reports in the English literature [3].

\subsection{Clinical Features}

Clinically, spontaneous retropharyngeal hematoma can present as a triad of features including superior mediastinal obstruction, anterior tracheal displacement and bruising on the neck within 48 hours with subsequent spreading to the chest wall [3]. However, in cases with no history of trauma, early diagnosis in an emergency department may be challenging because of non-specific symptoms, such as neck swelling or refused to feed and respiratory distress, especially when a hematoma is huge in a retropharyngeal space. Hematomas are assumed to expand within this loose areolar tissue, which may delay symptoms for at least 2 - 3 hours and possibly cause death. When dyspnea develops due to expansion of a hematoma, procedures such as a tracheostomy or tracheal intubation are required to secure the airway. Any delay in diagnosis can lead to complications like hypoxic in- 
jury to the brain with grave prognosis and even death. Computed tomography (CT) and MRI are used to diagnose a retropharyngeal hematoma, and close observation of prevertebral soft tissue width in lateral cervical spine radiograph is necessary.

\subsection{Differential Diagnosis}

Differential diagnosis of retropharyngeal hematoma includes retropharyngeal tendinitis and retropharyngeal infection. Retropharyngeal tendinitis is due to calcific deposits within the tendons of the longus colli muscles as in calcific deposits in calcific tendinitis of the shoulder. Radiographic findings are characteristically amorphous calcification localized anterior to C1 with associated swelling of the prevertebral (or retropharyngeal) soft tissues from C1 through C4 [3]. Clinical features are sudden onset of severe pain in the neck and throat aggravated by swallowing and movement of the head; this may be associated with mild fever and elevation of ESR. Pain reaches a maximum within $2-5$ days then gradually subsides, usually completely within 1 - 2 weeks. Initial clinical and radiologic findings in this case misled our diagnostic impression towards retropharyngeal abscess. Clinical findings of retropharyngeal infection include acute to subacute onset of neck pain, dysphagia, or odynophagia; mildly elevated WBC count; and low-grade fever. ESR may be mildly elevated, and there may be a recent history of upper respiratory infection or minor trauma to the head or neck [3]. Careful history taking, checking for signs of fever, and close follow-up of laboratory tests are important in differential diagnosis of retropharyngeal infection. Additional CT and MRI can also be helpful.

\subsection{Treatment}

Treatment of retropharygeal hematoma is basically to secure the airway and evacuate the hematoma. In cases involving large hematomas, first we secure the airway by orotracheal intubation and close monitoring of the vitals followed by surgical drainage in OT. Muñoz et al. [3] reported that in spontaneously developed hematoma, nearly complete absorption was observed after 1 week. However, in our case, symptoms gradually worsened and the patient went in severe respiratory distress; therefore, we went for immediate surgical drainage in OT under general anaesthesia to minimize the chances of aspiration.

\section{Conclusion}

Retropharyngeal hematoma is a life threatening disease. It is important to be aware of this unusual condition with its distinct presentation. Although surgical intervention is resisted unless a treatable aetiological factor is found or airway compromise occurs, any delay in the diagnosis and management can prove fatal. Large hematomas compressing the airway need airway to be secured and prompt surgical drainage, and others may resolve with conservative management.

\section{References}

[1] O’Donnell, J.J., Birkinshaw, R. and Harte, B. (1997) Mechanical Airway Obstruction Secondary to Retropharyngeal Haematoma. European Journal of Emergency Medicine, 4, 166-168. http://dx.doi.org/10.1097/00063110-199709000-00010

[2] Nurata, H., Yilmaz, M.B., Borcek, A.O., et al. (2012) Retropharyngeal Hematoma Secondary to Whiplash Injury in Childhood: A Case Report. Turkish Neurosurgery, 22, 521-523.

[3] Sang, S.K., et al. (2010) Spontaneous Retropharyngeal Hematoma-A Case Report. Korean Journal of Pain, 23, 211214. http://dx.doi.org/10.3344/kjp.2010.23.3.211 